\title{
Thyroid Hormone Replacement Therapy Is Associated With Longer Overall Survival in Patients With Resectable Gastroesophageal Cancer - a Retrospective Single Center Analysis
}

Hannah Christina Puhr

Medical University of Vienna: Medizinische Universitat Wien

Thorsten J Reiter

Medical University of Vienna: Medizinische Universitat Wien

Mohamed El-Mahrouk

Medical University of Vienna: Medizinische Universitat Wien

\section{Lena Saliternig}

Medical University of Vienna: Medizinische Universitat Wien

\section{Peter Wolf}

Medical University of Vienna: Medizinische Universitat Wien

Maximilian J Mair

Medical University of Vienna: Medizinische Universitat Wien

\section{Ariane Steindl}

Medical University of Vienna: Medizinische Universitat Wien

\section{Matthias Paireder}

Medical University of Vienna: Medizinische Universitat Wien

\section{Reza Asari}

Medical University of Vienna: Medizinische Universitat Wien

\section{Sebastian F Schoppmann}

Medical University of Vienna: Medizinische Universitat Wien

\section{Anna S Berghoff}

Medical University of Vienna: Medizinische Universitat Wien

\section{Matthias Preusser}

Medical University of Vienna: Medizinische Universitat Wien Ayseguel Ilhan-Mutlu ( $\square$ aysegul.ilhan@meduniwien.ac.at ) Medizinische Universitat Wien https://orcid.org/0000-0001-8291-0873

\section{Research Article}


Keywords: Gastrointestinal Neoplasms, Stomach Neoplasms, Esophageal Neoplasms, Thyroid gland, Thyroid diseases

Posted Date: May 5th, 2021

DOI: https://doi.org/10.21203/rs.3.rs-469310/v1

License: (c) (1) This work is licensed under a Creative Commons Attribution 4.0 International License. Read Full License 


\section{Abstract}

Purpose: As thyroid hormones modulate proliferative pathways, it is surmised that they can be associated with cancer development. Since the potential association of gastroesophageal cancer and thyroid disorders has not been addressed so far, the aim of this study was to investigate the association of thyroid hormone parameters with the outcome of these patients, so novel prognostic and even potentially therapeutic markers can be defined.

Methods: Clinical and endocrinological parameters of patients with resectable gastroesophageal cancer treated between 1990 and 2018 at the Vienna General Hospital, Austria including history of endocrinological disorders and laboratory analyses of thyroid hormones at first cancer diagnosis were investigated and correlated with the overall survival (OS).

Results: In a total of 865 patients, a tendency towards prolonged OS in hypothyroid patients (euthyroid, $n=647$ : median OS 29.7 months; hyperthyroid, $n=50: 23.1$ months; hypothyroid, $n=70: 47.9$ months; $p=0.069$ ) as well as a significant positive correlation of thyroid hormone replacement therapy with the OS was observed (without, $n=53$ : median OS 30.6 months; with, $n=67: 51.3$ months; $p=0.017$ ). Furthermore, triiodothyronine (T3) levels were also associated with the OS (median OS within limit of normal: 23.4, above: 32.4 , below: 9.6 months; $p=0.045$ ).

Conclusion: Thyroid disorders and their therapeutic interventions might be associated with the OS in patients with resectable gastroesophageal cancer. As data on the correlation of these parameters is scarce, this study proposes an important impulse for further analyses concerning the association of thyroid hormones with the outcome in patients with gastroesophageal tumors.

\section{Background}

Cancers of the upper gastrointestinal (GI) tract including gastric, esophageal and gastroesophageal junction (GEJ) cancer are among the most common and deadly malignant diseases worldwide (Bray et al. 2018). Survival in these patients is generally poor and reliable prognostic markers are scarce, especially in European cohorts (Feng et al. 2019). Given that clinical outcomes have been found to vary substantially even within the same stage groups, new prognostic tools are needed. As thyroid hormones modulate proliferative and angiogenic pathways, it is surmised that they may be associated with cancer development and progression (Brent 2012; Hercbergs et al. 2010; Liu et al. 2009). Furthermore, the loss of normal functions of thyroid receptors might also play an important role as a contributor to cancer development as well as metastasis (Moeller and Fuhrer 2013). However, data on the association of thyroid hormones and tumors, especially in the Gl tract, are scarce and to the best of our knowledge there currently is no data on the association of thyroid hormones and resectable gastroesophageal cancer. So far, we already investigated the potential survival correlation of thyroid hormones in advanced and metastatic gastroesophageal cancer patients and showed that elevated free thyroxine levels are associated with poorer OS in this patient cohort (Puhr et al. 2020). Thus, the aim of this study was to 
investigate the association of thyroid hormone parameters with the outcome of patients with resectable gastroesophageal tumors, so potential, novel prognostic markers can be defined.

\section{Materials And Methods Study design}

This analysis was a single center retrospective study assessing the prognostic association of the thyroid hormone status at the time of first diagnosis as well as patients' history of thyroid disorders and hormone replacement therapy with the course of disease of patients with resectable upper $\mathrm{Gl}$ cancers.

\section{Patients recruitment}

All patients, who had a histologically proven upper GI cancer and were either diagnosed and/or treated at the Department of Medicine I - Division of Oncology at the General Hospital of Vienna between 1990 and 2018 were included in this analysis. All included patients underwent tumor staging prior to therapy according to the local hospital standard, including history taking, physical examination, routine hematologic tests, upper gastrointestinal endoscopy with histological biopsy and CT of the chest and abdomen. Patients were treated according to the individual decision of an interdisciplinary tumor board, which ensured the best possible treatment according to the respective standard of knowledge at the time of diagnosis.

Patients were followed up until death according to the hospital or public records or loss to follow-up.

Patients under the age of 18 at the time of diagnosis were excluded as well as patients who were only diagnosed in the General Hospital of Vienna but subsequently treated exclusively in other health institutions.

\section{Data recruitment}

Clinical information including patient demographics, therapy regimens, thyroid hormones and survival outcome was obtained from hospital chart data. We evaluated endocrinological parameters including thyroid hormones including thyroid-stimulating hormone (TSH), triiodothyronine (T3), thyroxine (T4), free triiodothyronine (fT3) and free thyroxine (fT4). Normal limits were considered to be: TSH 0.44-3.77 $\mu \mathrm{U} / \mathrm{ml}$, T3 $0.8-1.8 \mathrm{ng} / \mathrm{ml}, \mathrm{T} 458-124 \mathrm{ng} / \mathrm{ml}$, fT4 0.76-1.66 ng/dl and fT3 2.15-4.12 pg/ml. All parameters were determined using standard assays and procedures according to hospital routine.

Thyroid disorders were defined using blood tests including TSH as well as fT4. TSH levels lower than the normal range of $0.44 \mu \mathrm{U} / \mathrm{ml}$ indicate hyperthyroidism and TSH levels higher than the normal range of $3.77 \mu \mathrm{U} / \mathrm{ml}$ indicate hypothyroidism. Whether the dysfunction is clinically relevant is determined by thyroxine levels. If TSH levels are out of normal range, but thyroxine levels are within the normal range the thyroid disorder is classified as subclinical. If TSH is lower and thyroxine is higher than the normal limit, 
this indicates clinically relevant hyperthyroidism. If TSH is higher and thyroxine is lower than the normal limit, this indicates clinically relevant hypothyroidism.

\section{Data safety}

Personal data was consecutively numbered and pseudonymized within data collection. Only authorized personnel have access to our database. Clinical data was stored in a password protected Filemaker(C) database on a protected server of the Medical University of Vienna.

\section{Statistical analysis}

Chi-squared test was utilized for the analysis of the distribution of dichotomized variables. Due to the hypothesis generating design of the current study no correction for multiple testing was applied (Bender and Lange 2001). Patients without an event (death) were censored at the date that they were last known to be alive.

Differences between groups were assessed using the Chi square test, the Kruskal-Wallis test, the MannWhitney $U$ test and the Pearson correlation as appropriate.

OS was calculated from the date of the initial diagnosis to the death of the patient or the patient's last follow-up date. The date of death was evaluated either by hospital chart data or by the records from the Austrian statistical office "Statistics Austria". Analyses of the OS were done with Kaplan-Meier survival estimates with log-rank test and Cox regression. Using log-rank test following parameters were correlated with outcome: gender, body mass index, nicotine, alcohol, family history, localization of cancer, histology, treatment (neoadjuvant versus adjuvant), surgical resection, radiotherapy, known endocrinological disorders. Using Cox-regression analysis, following parameters were correlated with outcome: age, serum $\mathrm{TSH}$, serum ft3, serum ft4, serum T3, serum T4.

Furthermore, patients were split up into groups with hormone levels below, within or above the normal range and their group assignment was correlated with OS using log-rank tests.

Two-tailed $p$-values of $\leq 0.05$ were considered to be statistically significant.

\section{Results}

\section{Patient and tumor characteristics}

Further analysis was performed on 865 patients with resectable gastroesophageal. Demographics and cancer specific characteristics are shown in Table 1. Twelve patients (1.4\%) were in a resectable setting, but the exact stage could not be identified due to missing data (i.e. no endoscopic ultrasound). The distribution of histological subtypes in relation to tumor localization is shown in Supplementary Fig. 1.

A history of a second cancer either before (100 patients, $11.6 \%$ ) or at the same time (64 patients, $7.4 \%$ ) of the diagnosis of gastroesophageal cancer was reported in around $19 \%$ of the patients. Concerning 
alcohol consumption, ninety-five patients were known alcoholics (11.0\%), whereas $310(35.8 \%)$ patients did not drink any alcohol and 311 (36.0\%) patients had a moderate consumption. Smoking was recorded in $414(47.9 \%)$ patients. Concerning treatment options, 455 (52.6\%) patients received chemotherapy either in an adjuvant, neoadjuvant or later on in a palliative setting. In total, 775 (89.6\%) patients received a surgical tumor removal, whereas radiation therapy was administered in $183(21.1 \%)$ patients.

\section{Overall survival in regard to patient and tumor characteristics}

At the time of data cut-off (December 2019) 684 (79.1\%) patients were dead, 181 (20.9\%) patients were either alive or loss to follow up. The median overall survival (OS) of the study population was 29.8 months $(95 \% \mathrm{Cl} 26.1-33.5)$.

The correlation of demographics and the baseline characteristics as well as characteristics of the malignancy with survival are shown as $p$ values in Table 1. The Kaplan-Meier figures can be seen in Supplementary Fig. 2. Older age is statistical significantly associated with shorter OS $(p<0.001 ; \mathrm{HR}$ 1.014). Furthermore, the tumor location $(p=0.004)$, the histology $(p=0.001)$ as well as the tumor stage ( $p$ $<0.001$ ) have a significant association with the OS.

Since patients were included into this study between 1990 and 2018, we analyzed weather the year of diagnosis had a statistically significant correlation with the OS. The OS increased statistically significantly throughout the years $(\mathbf{p}=\mathbf{0 . 0 2 5}, \mathrm{HR}=1.014,95 \% \mathrm{Cl} 1.002-1.027)$. However, in a multivariate analysis including stage and the year of diagnosis, stage remains statistically associated with the OS ( $\mathbf{p}$ $<0.001, H R=1.625)$, while the year of diagnosis is not $(p=0.485, H R=1.005)$.

\section{Thyroid parameters}

The median values of the evaluated thyroid parameters at the time of first diagnosis including TSH, T3, $\mathrm{T} 4$, $\mathrm{fT} 3$ and $\mathrm{fT} 4$ were within the normal range both in the overall and in separate cohorts based on the tumor stage. The results are shown in Table 2. Additionally, boxplots are available in Supplementary Fig. 3 to visualize these results in the overall cohort.

At the time of cancer diagnosis, the TSH levels of 209 (24.2\%) patients were available. In 7 patients the TSH levels were above the normal limit, suggesting hypothyroidism, and in 26 patients the levels were below the normal limit, suggesting hyperthyroidism.

In addition, known thyroid disorders in the patients' history were evaluated. In 50 (5.8\%) patients hyperthyroidism and in $70(8.1 \%)$ hypothyroidism was recorded. Six hundred and forty-seven $(74.8 \%)$ patients did not have a known thyroid disorder at the time of cancer diagnosis. In 98 (11.3\%) patients no data on thyroid disorders was available. Some patients had a history of subclinical thyroid disorders, which is shown in Supplementary Fig. 4.

In regard to tumor stages, the distribution of thyroid disorders is shown in Supplementary Fig. $\mathbf{5}$. 
Concerning therapies for thyroid disorders 67 (7.7\%) patients were taking thyroid hormone substitution therapy at the time of first cancer diagnosis and $41(4.7 \%)$ patients had had a thyroidectomy. Of these 67 patients taking thyroid replacement therapy 56 (83.6\%) had a history of hypothyroidism and $11(16.4 \%)$ had a history of hyperthyroidism (taking thyroid replacement therapy after complete thyroidectomy).

\section{Thyroid hormones and their correlation with the overall survival}

Concerning thyroid hormone levels, neither TSH nor T3, T4, fT3 or fT4 levels did not significantly correlate with the survival outcome, neither in the overall cohort nor in separate cohorts based on the tumor stage. These results are shown in Table 2.

Furthermore, we analyzed whether the values were within, above or below the limit of normal and correlated them with the OS. In the overall cohort T3 (median OS within the normal limit, $n=53: 23.4$ months, $95 \%$ Cl 3.9-42.9; above the normal limit, $n=1: 32.4$ months; below the normal limit, $n=6: 9.6$ months, $95 \% \mathrm{Cl} 7.1-12.1 ; \mathbf{p}=\mathbf{0 . 0 4 5}$ ) was statistical significantly associated with the OS. However, TSH (median OS within the normal limit, $\mathrm{n}=176$ : 26.0 months, $95 \% \mathrm{Cl} 17.0-35.0$; above the normal limit, $\mathrm{n}=7$ : 47.9 months, $95 \% \mathrm{Cl}$ 24.7-71.1; below the normal limit, $\mathrm{n}=26: 13.0$ months, $95 \% \mathrm{Cl} 4.9-21.1 ; \mathrm{p}=0.455$ ), T4 (median OS within the normal limit, $\mathrm{n}=82: 21.7$ months, $95 \% \mathrm{Cl}$ 10.0-33.4; below the normal limit, $\mathrm{n}=$ 7: 42.5 months, $95 \% \mathrm{Cl} 0.7-84.3 ; p=0.427$ ), fT3 (median OS within the normal limit, $n=33: 22.0$ months, 95\% $\mathrm{Cl}$ 8.2-35.8; above the normal limit, $n=3$ : 6.3 months, below the normal limit, $n=2: 19.7$ months; $p=$ 0.922 ) and fT 4 (within the normal limit, $n=72$; above the normal limit, $n=7$; below the normal limit, $n=1$; $p=0.968$; all cases censored) were not. These results are shown in Fig. 1.

\section{Thyroid diseases and their correlation with the overall survival}

The overall survival of patients with a history of thyroid disorders did not differ significantly from euthyroid patients, neither considering the overall cohort (overall cohort: Euthyroid, $n=647$ : median OS 29.7 months; 95\% Cl 26.0-33.4; Hyperthyroid, $\mathrm{n}=50$ : median OS 23.1 months; $95 \% \mathrm{Cl}$ 10.2-36.0; Hypothyroid, $n=70$ : median OS 47.9 months; $95 \% \mathrm{Cl} 20.1-75.7 ; p=0.069$ ) nor separate cohorts based on stages (stage I: Euthyroid, $n=121$ : median OS 82.8 months; $95 \%$ Cl 58.2-107.4; Hyperthyroid, $n=6$ : median OS 60.3 months; $95 \%$ Cl 0-156.6; Hypothyroid, $n=13$ : median OS 221.4 months; $95 \%$ Cl 110.9331.9; $p=0.147$; stage II: Euthyroid, $n=198$ : median OS 39.4 months; $95 \% \mathrm{Cl} 31.7-47.1 ;$ Hyperthyroid, $n=$ 17: median OS 36.5 months; $95 \% \mathrm{Cl} 10.7-62.3$; Hypothyroid, $\mathrm{n}=21$ : median $0 \mathrm{~S} 65.0$ months; $95 \% \mathrm{Cl}$ 37.0-93.1; $p=0.429$; stage III: Euthyroid, $n=320$ : median OS 19.6 months; $95 \% \mathrm{Cl} 17.1-22.1$; Hyperthyroid, $\mathrm{n}=26$ : median OS 18.0 months; $95 \% \mathrm{Cl}$ 6.6-29.4; Hypothyroid, $\mathrm{n}=35$ : median OS 26.7 months; $95 \% \mathrm{Cl} 15.8-37.6 ; p=0.259)$. However, both in the overall as well as in the separated cohorts a tendency that hypothyroid patients have a longer OS could be observed. This result is shown in Fig. 2.

The use of thyroid replacement therapy was associated with a statistically significant longer overall survival comparing patients with thyroid disorders in the overall cohort (without, $n=53$ : median 0 S 30.6 
months; $95 \% \mathrm{Cl} 12.9-48.3$; with, $\mathrm{n}=67$ : median OS 51.3 months; $95 \% \mathrm{Cl} 15.2-87.4 ; \mathrm{p}=0.017$ ). The same tendencies could be described in stage I (without, $n=5$ : median OS 60.3 months, $95 \%$ Cl 0-124.1; with, $n=$ 14: median OS 156.1 months, $95 \% \mathrm{Cl} 63.1-249.1 ; p=0.109$ ), stage II (without, $\mathrm{n}=18$ : median OS 38.6 months; $95 \% \mathrm{Cl} 20.3-56.9$; with, $\mathrm{n}=20$ : median OS 65.0 months; $95 \% \mathrm{Cl} 4.9-125.1 ; \mathrm{p}=0.548$ ) and stage III cohorts (without, $\mathrm{n}=29$ : median OS 18.6 months; $95 \% \mathrm{Cl} 10.1-27.1$; with, $\mathrm{n}=32$ : median $0 \mathrm{~S} 24.9$ months; $95 \% \mathrm{Cl} 14.7-35.1 ; \mathrm{p}=0.230$ ). These results are visualized with a Kaplan-Maier-curve in Fig. 2.

\section{Multivariate analysis}

All parameters, that were associated statically significantly with the OS in the univariate analysis, were included into the multivariate analysis (age, histology, tumor location, stage, thyroid replacement therapy, T3 levels according to normal limits). Tumor location $(p=0.15, H R=4.228)$, age at first diagnosis $(p=$ $\mathbf{0 . 0 0 2}$, HR $=1.231)$ and thyroid replacement treatment $(\mathbf{p}=\mathbf{0 . 0 1 5}, \mathrm{HR}=0.073)$ were still statically significant in the multivariate analysis, while stage $(p=0.557, H R=1.746)$, histology $(p=0.245, H R=$ $0.243)$ and T3 levels $(p=0.122, H R=2.368)$ were not.

\section{Discussion}

Gastroesophageal cancer is a widespread disease and accounts for a large portion of the global cancer mortality (Bray et al. 2018). Survival in these patients is poor and little is known about reliable prognostic markers. Since thyroid hormones have been found to convey proliferative and pro-angiogenic effects, it is surmised that they might also play a role in tumor development and progression (Hercbergs et al. 2010; Mousa et al. 2014). Thus, the aim of this study was to evaluate the association of thyroid hormones with the outcome in resectable gastroesophageal cancer patients.

\section{Demographic and tumor characteristics}

Cancers of the upper GI tract are known to be among the leading causes of global cancer mortality. While the diseases still affects more males than females (Ferlay et al. 2019), in recent decades a shift from squamous cell carcinomas to adenocarcinomas has been observed (Edgren et al. 2013). The conjecture behind this shift is, that changes in lifestyle in the Western world might effect the tumor incidence, e.g. increased prevalence of obesity, increased prevalence of gastroesophageal reflux disease, decline in the prevalence of $\mathrm{H}$. pylori colonization and improved food processing (Pandeya et al. 2010; Vaughan et al. 1995; Wheeler and Reed 2012). The results of these combined trends are also reflected in the data obtained in this study. Hence, we observed i) almost equal number of gastroesophageal junction (32.5\%) and gastric tumors (39.9\%), which is opposite to the Asian populations, where the gastric cases are in majority; ii) $70.2 \%$ of the included patients in this study were male and iii) the majority $(78.7 \%)$ of the observed carcinomas were adenocarcinomas. Furthermore the observed median age of the included patients of 64 years falls within the lines of the already known epidemiology seen in the Western world (Wheeler and Reed 2012). Thus, our study population is a large European cohort, that is representable and fits current data. 


\section{Thyroid hormone status}

For over a century, the association of thyroid hormones and malignant diseases has been suggested, yet it is still a widely discussed topic of grave scientific and clinical interest (Beatson 1896). Although, several in vitro and in vivo studies investigated the influence of thyroid parameters on upper GI cancers, clinical data on the subject is still scarce. So far, we have already discussed the association of elevated fT4 levels with poorer overall survival of patients with gastroesophageal cancer in advanced stages (Puhr et al. 2020). However, to our best knowledge there are no studies discussing this issue in resectable settings. Thus, the obtained data of this analysis shed some new light on this relationship and is an important impulse for further investigations.

Although, no statistically significant difference could be identified comparing the thyroid status in the patients' history with the OS, there was a clear tendency toward the association of hypothyroidism with a favorable and hyperthyroidism with a poor outcome (Fig. 2).

Most available literature agrees with this result (Berghoff et al. 2020; Cristofanilli et al. 2005; Hercbergs et al. 2010) and this finding supports the hypothesis, that thyroid hormones might have some tumor promoting properties, possibly by some non-canonical pathway (lishi et al. 1993; Liu et al. 2009).

Furthermore, the prevalence of known hyperthyroidism in our study cohort (5.7\%) is exceedingly high compared to epidemiological prevalence in literature (Vanderpump 2011). This finding underlines the possible role that thyroid hormones play in carcinogenesis (Piekiełko-Witkowska 2013) and was also investigated by other study groups (Kandemir et al. 2005).

In addition, thyroid hormone substitution was statistically significantly associated with the OS in our study cohort (Fig. 2). It is of note, that most research on the influence of thyroid hormone replacement therapy on tumor-progression has been conducted in patients who developed hypothyroidism under cancer-specific therapy and none has yet been conducted in gastroesophageal cancer to the best knowledge of the authors. Since thyroid replacement therapy is one of the most commonly prescribed drugs in Western countries (Mitchell et al. 2009), this finding is of high clinical interest. The rationale behind these results might be the different components of thyroid supplements and physiological thyroid hormones (Biondi and Wartofsky 2014).

Levothyroxine, which is the most commonly used thyroid supplement, is a synthetically generated substitute of T4 and thereby does not include T3. Thus, the serum levels of T4 might be higher in patients with replacement therapy, than in normal individuals, and the supplementation may not result in appropriate serum T3 concentrations (Mortoglou and Candiloros 2004). The compound of the supplement might be of high clinical interest, as T3 is discussed to be a more potent promoter of carcinogenesis due to its role as the key regulator of essential cellular processes including proliferation, differentiation, apoptosis and metabolism (Krashin et al. 2019). Thus, levothyroxine is widely discussed to be associated with a reduced risk of cancer. The association with a statistically significantly reduced relative risk of colorectal cancer was recently described (Rennert et al. 2010). However, this phenomenon 
has never been addressed in gastroesophageal cancer and other studies even suggest an association with increased cancer risk (Wu et al. 2018). Therefore, the results of this study are of grave scientific interest and give an important impulse to further analyze the association of thyroid replacement therapy with upper gastrointestinal cancer.

Furthermore, when we analyzed whether the thyroid hormone values were within, above or below the limit of normal a statistically significant difference in median OS could be observed for different T3 levels in the overall cohort. This correlation has also been described by several other research groups in different cancer entities (Czarnecka et al. 2016; Gao et al. 2018; Tosovic et al. 2014) and might also be associated with the nonthyroidal illness syndrome (NTIS), which was recently published to be connected with malignant diseases (Hercbergs et al. 2018).

However, there were only few patients with elevated or decreased levels and therefore these results might not be representable. This might be due to the timepoint of our measurements being the time of first cancer diagnosis, when most patients with a history of thyroid disease were already treated and therefore the levels were mostly within the normal limits.

Further investigation of the role of these parameters as prognostic markers needs to be conducted in the form of prospective studies in order to minimize missing values and potential arising biases.

\section{Strengths and limitations}

Strengths and limitations of the analysis need to be addressed. The homogenous study population represents a large European cohort with resectable upper gastrointestinal cancer. The best possible treatment for each patient was ensured by the individual decision of an interdisciplinary tumor board according to the respective standard of knowledge at the time of diagnosis and all patients were followed up regularly.

As this study was conducted retrospectively, the main limitation is missing data values. Due to obligatory and standardized laboratory monitoring as well as history taking at the Vienna General Hospital, most results were retrievable from the medical records. Still missing endocrinological parameters are listed in Supplementary table 1. Thus, to minimize missing data values and confirm the results of our analysis a prospective study should be conducted. Furthermore, a more detailed examination of laboratory values in a prospective setting might be able to identify the underlying cause of the observed associations. Moreover, longitudinal measurements of endocrinological parameters could give more detail about the influence of thyroid hormones throughout disease progression and should therefore be conducted in further studies.

\section{Conclusion}

In conclusion, the results of this retrospective single center analysis indicate, that a history of hypothyroidism as well as thyroid hormone replacement therapy with levothyroxine might be associated with a beneficial outcome in patients with resectable gastroesophageal cancer. These results are in line 
with our results in patients with advanced stages and other studies in various other cancer entities. The role of thyroid hormones in carcinogenesis is still not fully understood and a topic of grave scientific interest and potential clinical relevance. Thus, our results are an important impulse for further prospective studies concerning the association of thyroid hormones with the outcome in patients with tumors of the upper gastrointestinal tract.

\section{Abbreviations}

fT3 - free triiodothyronine, fT4 - free thyroxine, GEJ - gastroesophageal junction, GI - gastrointestinal, HR hazard ratio, NTIS - nonthyroidal illness syndrome, OS - overall survival, T3 - triiodothyronine, T4 thyroxine, TSH - thyroid-stimulating hormone

\section{Declarations}

\section{Funding}

The authors gratefully acknowledge Medical University of Vienna's core funding to the Department of Medicine I.

\section{Authors' contributions and consent for publication}

The manuscript has been read and approved by all authors. All authors contributed to the preparation of the manuscript significantly and are in agreement with the content of the manuscript. All authors were involved in creating the study design and concept. All authors confirm that the manuscript is original and has not been submitted elsewhere.

\section{Conflicts of interest}

HC.P. has received travel support from Eli Lilly, MSD, Novartis, Pfizer and Roche.

AS.B. has received research support from Daiichi Sankyo and Roche, honoraria for lectures, consultation or advisory board participation from Roche, Bristol-Meyers Squibb, Merck, Daiichi Sankyo as well as travel support from Roche, Amgen, Daiichi Sankyo and AbbVie. M.P. has received honoraria for lectures, consultation or advisory board participation from the following for-profit companies: Bayer, Bristol-Myers Squibb, Novartis, Gerson Lehrman Group (GLG), CMC Contrast, GlaxoSmithKline, Mundipharma, Roche, BMJ Journals, MedMedia, Astra Zeneca, AbbVie, Lilly, Medahead, Daiichi Sankyo, Sanofi, Merck Sharp \& Dome, Tocagen. The following for-profit companies have supported clinical trials and contracted research conducted by M.P. with payments made to his institution: Böhringer-Ingelheim, Bristol-Myers Squibb, Roche, Daiichi Sankyo, Merck Sharp \& Dome, Novocure, GlaxoSmithKline, AbbVie.

A.I-M. participated in advisory boards from MSD, BMS and Servier, received lecture honoraria from Eli Lilly, MSD and Servier, is the local PI for clinical trials sponsored by BMS and Roche and received travel support from BMS and Roche. 
Other authors have nothing to declare.

\section{Ethics approval}

All procedures were in accordance with the ethical standards of the responsible committee on human experimentation and with the Helsinki Declaration of 1964 and later versions. Due to the retrospective design no separate informed consent was necessary in the scope of this study. The study was approved by the ethics committee of the Medical University of Vienna (reference number: 1211/2020).

\section{Availability of data and material}

The data that support the findings of this study are available from the corresponding author, A.I-M., upon reasonable request.

\section{Acknowledgements}

The authors gratefully acknowledge Medical University of Vienna's core funding to the Department of Medicine I. This study was performed within the PhD thesis of HC.P. with the title "Immunological, genetic and prognostic factors related to outcome in patients with upper gastrointestinal tract cancer" in the Clinical \& Experimental Oncology program at the Medical University of Vienna. This analysis was presented as a poster at the European Society of Medical Oncology 2020 Annual Congress.

\section{Novelty \& Impact statement}

Thyroid hormones are surmised to can be associated with cancer. We analyzed patients with resectable gastroesophageal cancer and demonstrated the association of thyroid hormone replacement therapy with the overall survival.

\section{References}

1. Beatson GT (1896) On the Treatment of Inoperable Cases of Carcinoma of the Mamma: Suggestions for a New Method of Treatment, with Illustrative Cases Trans Med Chir Soc Edinb 15:153-179

2. Bender R, Lange S (2001) Adjusting for multiple testing-when and how? J Clin Epidemiol 54:343-349 doi:10.1016/s0895-4356(00)00314-0

3. Berghoff AS et al. (2020) Hypothyroidism correlates with favourable survival prognosis in patients with brain metastatic cancer European Journal of Cancer 135:150-158 doi:https://doi.org/10.1016/j.ejca.2020.05.011

4. Biondi B, Wartofsky L (2014) Treatment with thyroid hormone Endocr Rev 35:433-512 doi:10.1210/er.2013-1083

5. Bray F et al. (2018) Global cancer statistics 2018: GLOBOCAN estimates of incidence and mortality worldwide for 36 cancers in 185 countries CA Cancer J Clin 68:394-424 doi:10.3322/caac.21492 
6. Brent GA (2012) Mechanisms of thyroid hormone action The Journal of clinical investigation 122:3035-3043 doi:10.1172/jci60047

7. Cristofanilli $\mathrm{M}$ et al. (2005) Thyroid hormone and breast carcinoma. Primary hypothyroidism is associated with a reduced incidence of primary breast carcinoma Cancer 103:1122-1128 doi:10.1002/cncr.20881

8. Czarnecka AM et al. (2016) Triiodothyronine regulates cell growth and survival in renal cell cancer Int J Oncol 49:1666-1678 doi:10.3892/ijo.2016.3668

9. Edgren $\mathrm{G}$ et al. (2013) A global assessment of the oesophageal adenocarcinoma epidemic Gut 62:1406-1414 doi:10.1136/gutjnl-2012-302412

10. Feng Q et al. (2019) Prognostic Models for Predicting Overall Survival in Patients with Primary Gastric Cancer: A Systematic Review BioMed Research International 2019:5634598 doi:10.1155/2019/5634598

11. Ferlay J et al. (2019) Estimating the global cancer incidence and mortality in 2018: GLOBOCAN sources and methods Int J Cancer 144:1941-1953 doi:10.1002/ijc.31937

12. Gao R et al. (2018) Low T3 syndrome as a predictor of poor prognosis in chronic lymphocytic leukemia International Journal of Cancer 143:466-477 doi:10.1002/ijc.31327

13. Hercbergs A et al. (2018) Nonthyroidal Illness Syndrome and Thyroid Hormone Actions at Integrin avß3 The Journal of Clinical Endocrinology \& Metabolism 103:1291-1295 doi:10.1210/jc.201701939

14. Hercbergs AH et al. (2010) Thyroid hormones and cancer: clinical studies of hypothyroidism in oncology Current opinion in endocrinology, diabetes, and obesity 17:432-436 doi:10.1097/MED.0b013e32833d9710

15. lishi $\mathrm{H}$ et al. (1993) Enhancement by thyroxine of gastric carcinogenesis induced by N-methyl-N'nitro-N-nitrosoguanidine in Wistar rats British journal of cancer 68:515-518 doi:10.1038/bjc.1993.378

16. Kandemir EG et al. (2005) Gastric carcinoma and thyroid status J Int Med Res 33:222-227 doi:10.1177/147323000503300210

17. Krashin E et al. (2019) Thyroid Hormones and Cancer: A Comprehensive Review of Preclinical and Clinical Studies Front Endocrinol (Lausanne) 10:59-59 doi:10.3389/fendo.2019.00059

18. Liu R et al. (2009) Mechanism of cancer cell adaptation to metabolic stress: proteomics identification of a novel thyroid hormone-mediated gastric carcinogenic signaling pathway Mol Cell Proteomics 8:70-85 doi:10.1074/mcp.M800195-MCP200

19. Mitchell AL et al. (2009) Trends in thyroid hormone prescribing and consumption in the UK BMC Public Health 9:132 doi:10.1186/1471-2458-9-132

20. Moeller LC, Fuhrer D (2013) Thyroid hormone, thyroid hormone receptors, and cancer: a clinical perspective Endocr Relat Cancer 20:R19-29 doi:10.1530/ERC-12-0219

21. Mortoglou A, Candiloros $H$ (2004) The serum triiodothyronine to thyroxine (T3/T4) ratio in various thyroid disorders and after Levothyroxine replacement therapy Hormones (Athens) 3:120-126 
doi:10.14310/horm.2002.11120

22. Mousa SA et al. (2014) Modulation of angiogenesis by thyroid hormone and hormone analogues: implications for cancer management Angiogenesis 17:463-469 doi:10.1007/s10456-014-9418-5

23. Pandeya $\mathrm{N}$ et al. (2010) Gastro-oesophageal reflux symptoms and the risks of oesophageal cancer: are the effects modified by smoking, NSAIDs or acid suppressants? Gut 59:31-38 doi:10.1136/gut.2009.190827

24. Piekiełko-Witkowska A (2013) Molecular mechanism of thyroid hormone action in carcinogenesis Thyroid Res 6:A48-A48 doi:10.1186/1756-6614-6-S2-A48

25. Puhr HC et al. (2020) Elevated Free Thyroxine Levels Are Associated with Poorer Overall Survival in Patients with Gastroesophageal Cancer: A Retrospective Single Center Analysis Hormones \& cancer 11:42-51 doi:10.1007/s12672-019-00374-1

26. Rennert $\mathrm{G}$ et al. (2010) A case-control study of levothyroxine and the risk of colorectal cancer Journal of the National Cancer Institute 102:568-572 doi:10.1093/jnci/djq042

27. Tosovic A et al. (2014) T3 levels in relation to prognostic factors in breast cancer: a population-based prospective cohort study BMC Cancer 14:536 doi:10.1186/1471-2407-14-536

28. Vanderpump MP (2011) The epidemiology of thyroid disease Br Med Bull 99:39-51 doi:10.1093/bmb/ldr030

29. Vaughan TL et al. (1995) Obesity, alcohol, and tobacco as risk factors for cancers of the esophagus and gastric cardia: adenocarcinoma versus squamous cell carcinoma Cancer Epidemiol Biomarkers Prev 4:85-92

30. Wheeler JB, Reed CE (2012) Epidemiology of esophageal cancer Surg Clin North Am 92:1077-1087 doi:10.1016/j.suc.2012.07.008

31. Wu C-C et al. (2018) Levothyroxine use and the risk of breast cancer: a nation-wide population-based case-control study Archives of Gynecology and Obstetrics 298:389-396 doi:10.1007/s00404-0184837-y

\section{Tables}

Due to technical limitations, table 1 \& 2 PDF are only available as a download in the Supplemental Files section.

\section{Figures}



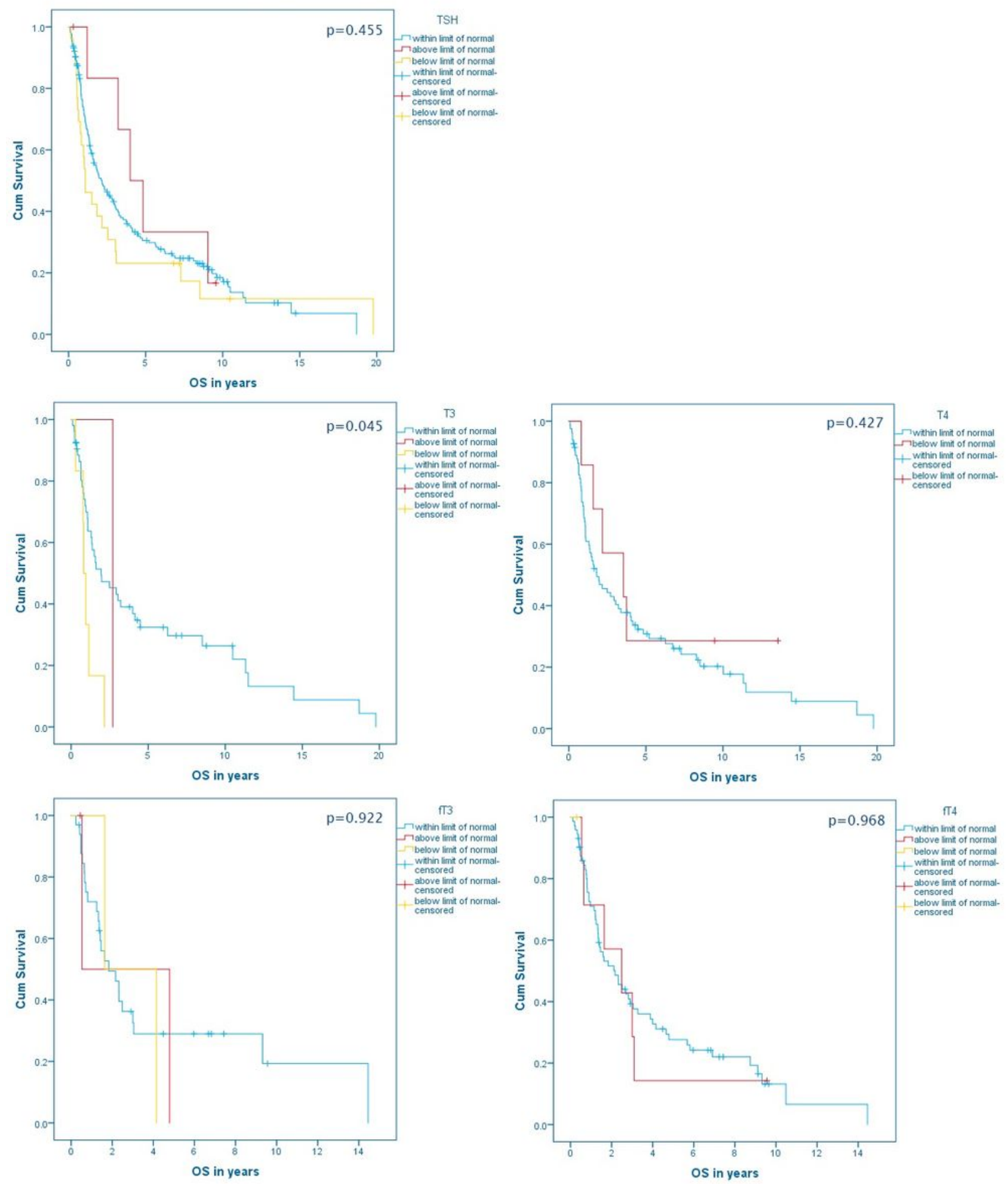

\section{Figure 1}

The correlation of thyroid hormone levels within, above and below normal limits with the overall survival in patients with resectable gastroesophageal cancer. 


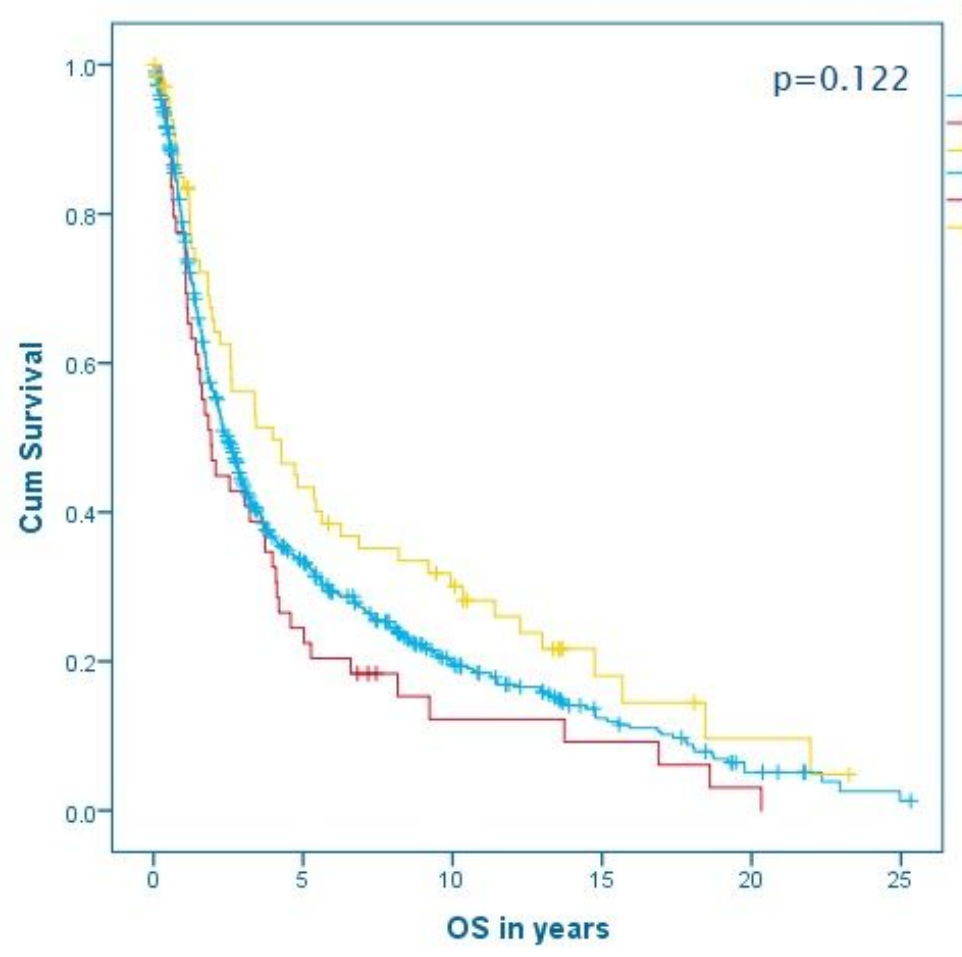
Thyroid hormone status
REuthyroid
ᄀHyperthyroid
Hypothyroid
4-Euthyroid-censored
+ Hyperthyroid-censored
Hypothyroid-censored

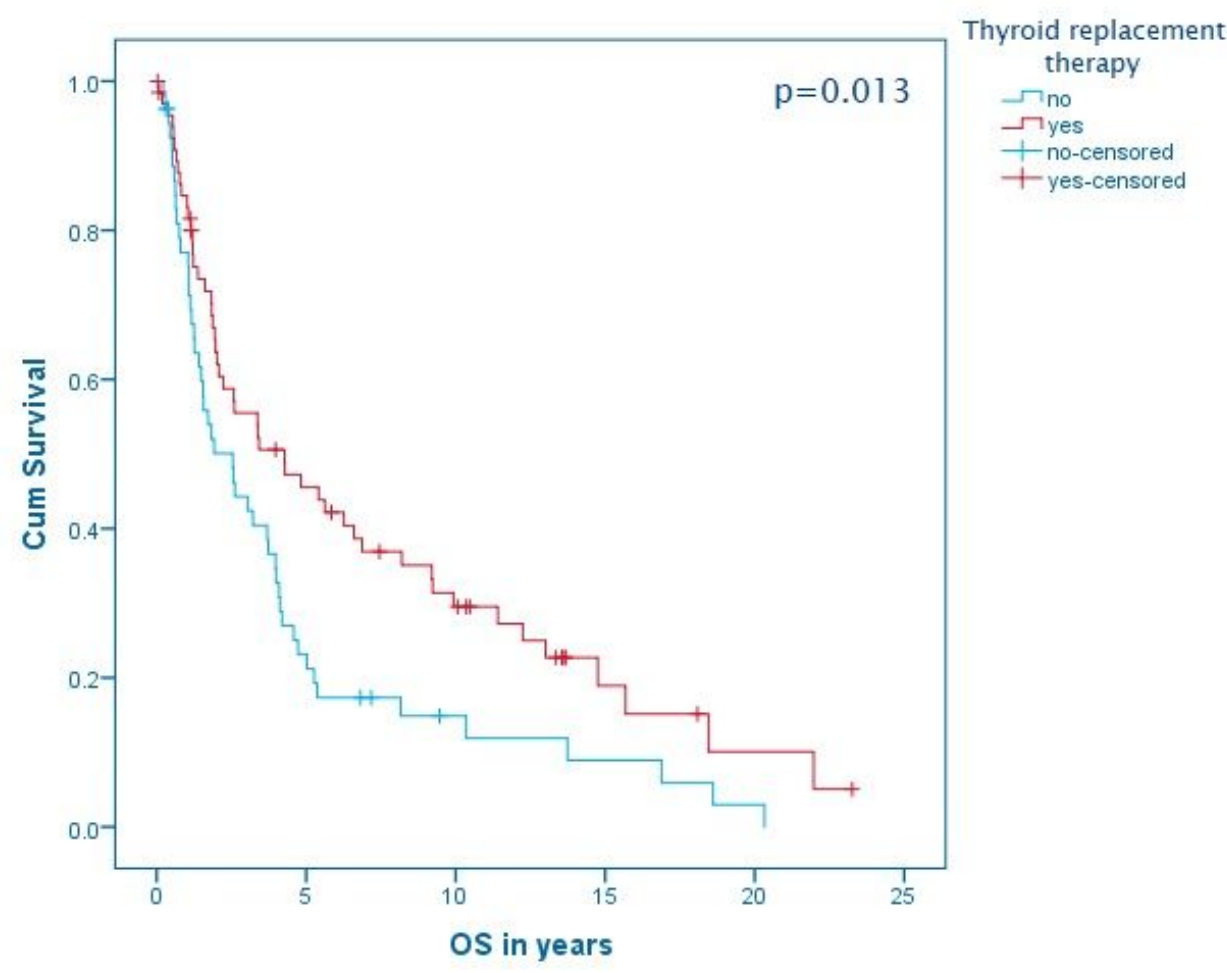

Figure 2

Kaplan Meier curves concerning the thyroid hormone status as well as thyroid replacement therapy.

\section{Supplementary Files}

This is a list of supplementary files associated with this preprint. Click to download. 
- Table1.pdf

- Table2.pdf

- Supplementaryfigure1.pdf

- Supplementaryfigure2.pdf

- Supplementaryfigure3.pdf

- Supplementaryfigure4.pdf

- Supplementaryfigure5.pdf

- Supplementarytable1.pdf 